\title{
75 \\ Oculomotor Problems Associated with the Blepharophimosis Syndrome
}

\author{
J. W. Walker and R. Collins
}

The Blepharophimosis syndrome consists of blepharophimosis or a narrowed palpebral aperture, ptosis, telecanthus and epicanthus inversus. It is inherited as autosomal dominant, but half of our cases occurred as fresh mutations. We have studied nearly 100 patients with this syndrome to assess the incidence of squint and amblyopia. 